Assurances et gestion des risques

Insurance and Risk Management

\title{
Droit à l'oubli pour les malades de cancers : un risque relatif pour les assurances
}

\section{Jérémy Picot}

Volume 84, numéro 3-4, décembre 2017

URI : https://id.erudit.org/iderudit/1043360ar

DOI : https://doi.org/10.7202/1043360ar

Aller au sommaire du numéro

\section{Éditeur(s)}

Faculté des sciences de l'administration, Université Laval

\section{ISSN}

1705-7299 (imprimé)

2371-4913 (numérique)

Découvrir la revue

\section{Citer ce document}

Picot, J. (2017). Droit à l'oubli pour les malades de cancers : un risque relatif pour les assurances. Assurances et gestion des risques / Insurance and Risk Management, 84(3-4), 153-163. https://doi.org/10.7202/1043360ar

\section{Résumé de l'article}

La signature de la convention AERAS en 2006 a été une avancée majeure en permettant l'accès aux crédits pour des milliers de malades et anciens malades. Aujourd'hui, le projet de loi santé prévoit la mise en place d'un droit à l'oubli pour les malades de cancers. Sa localisation et le pronostic moyen associé seront susceptibles de faire varier le délai permettant d'accéder à ce droit. Tandis que les compagnies d'assurance souhaitent voir l'obligation de déclaration conservée pendant 15 ans après le diagnostic, les associations de malades militent pour que ce délai soit réduit à 10 voire 5 ans, dans certains cas. En croisant les données épidémiologiques et les données bancaires, nous démontrerons que le nombre supplémentaire d'assurés potentiels engendré par la réduction de ce délai ne représenterait qu’une augmentation très marginale des risques couverts par les assureurs.
Tous droits réservés $\odot$ Faculté des sciences de l'administration, Université Laval, 2018
Ce document est protégé par la loi sur le droit d'auteur. L’utilisation des services d'Érudit (y compris la reproduction) est assujettie à sa politique d'utilisation que vous pouvez consulter en ligne.

https://apropos.erudit.org/fr/usagers/politique-dutilisation/ 


\section{DROIT À L'OUBLI POUR LES MALADES DE CANCERS: UN RISQUE RELATIF POUR LES ASSURANCES}

Jérémy PICOT1

\section{RESUMÉ}

La signature de la convention AERAS en 2006 a été une avancée majeure en permettant l'accès aux crédits pour des milliers de malades et anciens malades. Aujourd'hui, le projet de loi santé prévoit la mise en place d'un droit à l'oubli pour les malades de cancers. Sa localisation et le pronostic moyen associé seront susceptibles de faire varier le délai permettant d'accéder à ce droit. Tandis que les compagnies d'assurance souhaitent voir l'obligation de déclaration conservée pendant 15 ans après le diagnostic, les associations de malades militent pour que ce délai soit réduit à 10 voire 5 ans, dans certains cas. En croisant les données épidémiologiques et les données bancaires, nous démontrerons que le nombre supplémentaire d'assurés potentiels engendré par la réduction de ce délai ne représenterait qu'une augmentation très marginale des risques couverts par les assureurs.

MOTS CLÉS : Cancer - Droit à l'oubli - Assurance de prêts.

\section{INTRODUCTION}

L'assurance de prêt est souvent, en particulier dans le cadre du financement d'une opération immobilière, une condition indispensable à l'accord de l'organisme prêteur. Avant l'application de la convention AERAS $^{2}$ (s'Assurer et Emprunter avec un Risque Aggravé de Santé), en 2007 , les personnes souffrant ou ayant souffert de pathologies graves et/ou chroniques se retrouvaient ainsi, de fait, exclues de l'accès 
à ces crédits. Issue d'un long processus de négociations et de prises de consciences, cette convention nécessite, encore à l'heure actuelle, des améliorations et des mises en adéquation avec, entre autres, les progrès thérapeutiques.

Dans cette optique, en septembre 2015, le projet de "loi de santé" porté par Marisole Touraine ajoute à cette convention un principe de "droit à l'oubli». Cette disposition prend deux formes. D'une part, les anciens malades d'un cancer pourront ne pas déclarer cette pathologie, en respectant un certain délai, variable suivant la localisation du cancer, pour les personnes majeures au moment du diagnostic. D'autre part, ces délais feront l'objet de la mise en place d'une grille de référence précisant les localisations des cancers concernés et les autres pathologies, notamment chroniques, faisant l'objet de ce droit à l'oubli. Les associations de malades militent pour inciter à l'adoption d'une grille qui permettrait de raccourcir les périodes de déclaration obligatoire aux assurances pour les anciens malades de cancer. Ce délai, actuellement de 15 ans, pourrait être porté à 5 ans pour les cancers de bon pronostic (c'est-à-dire dont le taux de survie à 5 ans est supérieur à $80 \%$ ), d'une part, et à 10 ans, pour les autres cancers.

Pour ces personnes, cette disposition est une véritable aubaine. En effet, ce droit à l'oubli leur permettra d'accéder au crédit et surtout à l'assurance de celui-ci sans surprime et sans exclusion de garantie. Dans le cas contraire, à la non couverture du risque de décès ou d'invalidité en lien avec la pathologie dont ils sont ou ont été atteints, peut s'ajouter une majoration du taux de l'assurance emprunteur allant jusqu'à une augmentation de 1,4 point du taux effectif global (TEG). D'un point de vue social et éthique, l'enjeu est majeur. Qu'en est-il du point de vue des compagnies d'assurance? Le risque financier est-il aussi important pour elles?

L'objectif de l'étude est de réunir des données statistiques fournies par différents organismes nationaux (Les Hopitaux de Lyon, l'INCa ${ }^{3}$, la Fédération Française des Assureurs, l'Observatoire des Crédits aux Ménages, etc...). Leur croisement permettra de réaliser une estimation du nombre de personnes qui pourraient être potentiellement concernées par ce dispositif et de l'impact annuel que cette évolution aurait pour les assureurs.

Pour des raisons évidentes, la population de référence sera, pour les données bancaires, constituée des individus dont l'âge est inférieur à 55 ans et, par conséquent, dans la mesure où l'on cherche à prouver 
l'intérêt du droit à l'oubli à 5 ans, pour les données épidémiologiques, les personnes de moins de 50 ans lors du diagnostic d'un cancer de bon pronostic et de moins de 45 ans lors du diagnostic d'un autre type de cancer. Après avoir présenté les données bancaires et épidémiologiques, nous procèderons à leur analyse croisée afin d'évaluer l'importance du passage du droit à l'oubli de 15 ans à 5 ans pour les malades de cancers de bon pronostic. La dernière partie nous permettra de conclure.

\section{DESCRIPTION DES DONNÉES ÉTUDIÉES}

\subsection{Des ménages peu enclins à souscrire à un crédit immobilier}

L'Observatoire des Crédits aux Ménages (OCM) publie chaque année un état des lieux de l'ensemble des crédits détenus par les français. Le dernier rapport annuel [1], daté du 29 janvier 2015, tire les conclusions d'une enquête réalisée par TNS-SOFRES pour le compte de l'OCM et dresse le portrait des ménages français sous l'angle de leur relation au crédit.

Plus particulièrement, on s'aperçoit qu'en 2014, la part des ménages français déclarant envisager de demander un crédit immobilier est de $4,2 \%$.

Cette intention de souscrire à un crédit immobilier est relativement stable. Après avoir atteint trois pics en 2005, 2007 et 2008, elle a décru pour atteindre un minimum de $4 \%$ en 2011. Dans un passé plus récent, la tendance est à la stabilisation avec un taux de croissance annuel moyen de la part des ménages envisageant souscrire à un crédit immobilier de 1,6\% sur la période 2011 - 2014. Il est donc tout à fait acceptable de considérer que cette part restera stable dans les prochaines années.

Parmi les ménages exprimant un désir de contracter un crédit immobilier en novembre 2014, la part de ceux dont la personne de référence est âgée de moins de 55 ans est de $86,5 \%$. Ainsi, on peut conclure que les ménages dont le chef a moins de 55 ans et qui déclarent réfléchir à la demande d'un prêt immobilier représentent 3,63\% des ménages français en novembre 2014. 
Étant donnée la stabilité de ces intentions, on peut considérer qu'il sera possible dans la suite de ce rapport, d'évaluer le taux de ménages de moins de 55 ans ayant pour projet de contracter un prêt immobilier à un niveau contenu dans un intervalle compris entre 3,5 et $4 \%$ pour les années à venir.

\subsection{Montant moyen des prêts immobiliers}

Depuis 1978, l'Observatoire Permanent sur le Financement du Logement mène chaque année, en collaboration avec le CSA, une enquête auprès des établissements distributeurs de crédit sur le financement par emprunt des opérations immobilières effectuées par les ménages.

La 9ème édition de la synthèse des analyses de l'Observatoire crédit logement - CSA [2] nous apprend notamment que le montant moyen emprunté pour une opération immobilière quelconque est, en 2013, de l'ordre de 93,1 milliers d'euros. Si on considère uniquement les opérations d'acquisition neuves, le montant moyen des prêts dans le financement de ces opérations est de 158,2 milliers d'euros.

Nous croiserons ces données essentielles du secteur bancaire avec celle développées maintenant concernant les cancers de bon pronostic.

\section{UN SURCROIT MARGINAL DE DEMANDES DE CRÉDITS IMMOBILIERS}

\subsection{Données épidémiologiques: une excellente survie à 5 des cancers à relativiser}

Les cancers dits de bon pronostic sont ceux pour lesquels le taux de survie nette à 5 ans (c'est-à-dire la part de personnes non décédées en raison du cancer dont elles sont atteintes 5 ans après l'établissement du diagnostic) est supérieur à $80 \%$. Il s'agit des cancers du sein, de la prostate, des testicules, des lèvres, de la thyroïde, des LLC et des mélanomes.

On calcule également un taux de survie brute qui constitue la part des patients encore en vie 5 ans après le diagnostic de leur cancer quelle que soit la cause éventuelle des décès constatés. Ce taux brut est évidemment toujours plus élevé que la survie nette. Cependant, il sera également examiné chaque fois que ce sera possible tout au long de l'étude dans un souci de complétude des informations. 
Ainsi, l'Institut National du Cancer nous indique qu'en 2012, le nombre des diagnostics de cancers de bon pronostic s'élève à 19358 . Le détail des taux de survie (brute et nette) à 5 ans fourni par l'Institut de veille sanitaire, le Registre des cancers Francim, les Hôpitaux de Lyon et l'Institut National du cancer [3] pour chacune des catégories de cancer nous permet de réaliser des projections qui comptabilisent le nombre de personnes diagnostiquées en 2012 et qui seront encore en vie en 2017. On arriverait ainsi à un taux de survie nette à 5 ans de $88 \%$ (et une survie brute de $82 \%$ ) pour l'ensemble des ces individus. En d'autres termes, 17100 personnes de moins de 50 ans diagnostiquées en 2012 devraient être encore en vie en 2017.

Ceci étant dit, il est clair que ces personnes ne seront pas toutes des demandeurs de crédits immobiliers. C'est pour cette raison qu'il est intéressant de croiser ces résultats avec les chiffres des intentions de demandes de crédits immobiliers décrits précédemment.

\subsection{Passage du droit à l'oubli à 5 ans pour les cancers de bon pronostic: moins de 700 individus concernés par an}

Dans l'ensemble de la population, il apparaît qu'environ $36 \%$ des ménages sont endettés dans le cadre d'un prêt immobilier. Il est donc évident que parmi les personnes qui ont été diagnostiquées en 2012 et qui seront encore en vie en 2017 , un certain nombre d'entre elles auront déjà contracté un emprunt immobilier avant leur maladie (et appartiendront ainsi au $36 \%$ de ménages qui sont peu ou pas susceptibles de réaliser une nouvelle demande pour obtenir un tel emprunt). Il nous semble donc raisonnable de croiser de manière très simple ce nombre d'individus avec les données précédemment exposées en ce qui concerne la part des ménages de moins de 55 ans qui ont l'intention de souscrire à un crédit immobilier.

Pour mémoire, cette part s'élève à 3,63\% des ménages français en novembre 2014 et reste peu évolutive. Nous choisirons donc de réaliser une estimation du nombre maximum de crédits immobiliers potentiellement demandés en 2017 par des personnes de moins de 55 ans et ayant été diagnostiquées 5 ans auparavant atteintes d'un cancer de bon pronostic en se fondant sur ce taux de 3,63\% des ménages français.

En considérant la projection précédente, cela représenterait un total de 621 intentions de demandes de crédits immobiliers au maximum par an. Dans la mesure où on constate que le montant moyen emprunté pour une opération immobilière quelconque est, en 2013, de l'ordre de 93,2 milliers d'euros (montant moyen qui s'élève à 158,2 milliers d'euros, si on s'intéresse uniquement aux opérations d'acquisition 
neuves), le montant total des prêts demandés (en se fondant toujours sur une faible évolution de ces montants moyens) par les sujets de notre étude s'élèverait à 57,8 millions d'euros maximum chaque année.

Ces 57,8 millions d'euros de crédits supplémentaires ne représentent qu'une très faible augmentation (de l'ordre de 0,0008\%) lorsqu'on considère les 119600 millions d'euros prêtés au titre de crédits immobiliers en 2014.

\section{TABLEAU 1 Projection des demandes de crédits immobiliers en 2017 par les patients atteints d'un cancer de bon pronostic diagnostiqués en 2012}

\begin{tabular}{c|c|c|c|c|c|c}
\multicolumn{2}{c}{} & \multicolumn{2}{c}{$\begin{array}{c}\text { Base : crédit immobilier } \\
\text { moyen de } 93100 \text { euros }\end{array}$} & \multicolumn{2}{c}{$\begin{array}{c}\text { Base : crédit immobilier } \\
\text { moyen de } 158200 \text { euros }\end{array}$} \\
\cline { 2 - 7 } & $\begin{array}{c}\text { Nombre de } \\
\text { personnes } \\
\text { en vie 5 ans } \\
\text { après le } \\
\text { diagnostic }\end{array}$ & $\begin{array}{c}\text { Nombre } \\
\text { maximum } \\
\text { de crédits } \\
\text { immobiliers } \\
\text { demandés }\end{array}$ & $\begin{array}{c}\text { Montant total } \\
\text { maximum } \\
\text { de crédits } \\
\text { immobiliers } \\
\text { demandés, } \\
\text { milliers d'euros }\end{array}$ & $\begin{array}{c}\text { Pourcentage } \\
\text { d'augmentation } \\
\text { des crédits } \\
\text { immobiliers } \\
\text { accordés }\end{array}$ & $\begin{array}{c}\text { Montant total } \\
\text { maximum } \\
\text { de crédits } \\
\text { immobiliers } \\
\text { demandés, en } \\
\text { milliers d'euros }\end{array}$ & $\begin{array}{c}\text { Pourcentage } \\
\text { d'augmentation } \\
\text { des crédits } \\
\text { immobiliers } \\
\text { accordés }\end{array}$ \\
\hline $\begin{array}{c}\text { En survie } \\
\text { nette }\end{array}$ & 17099,48 & 621,22 & 57815,1 & $0,0005 \%$ & 98242,2 & $0,0008 \%$ \\
\hline $\begin{array}{c}\text { En survie } \\
\text { brute }\end{array}$ & 15892,45 & 577,37 & 53718,7 & $0,0004 \%$ & 91281,4 & $0,0008 \%$ \\
\hline
\end{tabular}

\subsection{Passage du droit à l'oubli à 10 ans pour les autres types de cancers}

L'institut de veille sanitaire fournit [6] les chiffres des cas incidents estimés par tranches d'âge en 2005 pour l'ensemble des cancers. On peut alors calculer le nombre de cancers de diagnostic intermédiaire ou mauvais diagnostiqués sur les individus qui auront l'âge d'emprunter 10 ans plus tard, en 2015. Ces individus appartiennent à la tranche d'âge 10 - 44 ans en 2015. Ils sont ainsi estimés à 8423 .

En appliquant les taux respectifs de survie brute fournis par l'Institut de veille sanitaire [3] à chacun des types de cancers de pronostic intermédiaire ou mauvais, on estime le nombre de survivants en 2015 à 4930 sur les 8423 diagnostiqués en 2005. Parmi eux, 3,63\%, soit 179 individus sont susceptibles de souscrire à un emprunt immobilier.

De la même manière que pour le passage du droit à l'oubli à 5 ans des cancers de bon pronostic, le passage à 10 ans pour les autres types de cancer ne produit donc pas un surcroît de demande de crédits 
immobiliers important. En effet, ces demandes représenteraient au maximum 16,66 millions d'euros, sur la base d'un crédit immobilier d'un montant moyen de 93,1 milliers d'euros. On arriverait donc à un impact encore moins important du passage à 10 ans du droit à l'oubli pour les cancers de pronostic intermédiaire ou mauvais.

TABLEAU 2 Nombre 2005 de cancers de diagnostic intermédiaire ou mauvais, survie nette à 5 ans et projection du nombre de survivants en 2015

\begin{tabular}{|c|c|c|c|c|c|c|c|}
\hline & $\begin{array}{l}\text { Effectif } \\
\text { Hommes }\end{array}$ & $\begin{array}{l}\text { Effectif } \\
\text { Femmes }\end{array}$ & $\begin{array}{l}\text { Survie nette } \\
\text { à } 5 \text { ans } \\
\text { Hommes }\end{array}$ & $\begin{array}{c}\text { Survie nette } \\
\text { à } 5 \text { ans } \\
\text { Femmes }\end{array}$ & $\begin{array}{c}\text { Projections } \\
\text { survivants } \\
\text { Hommes }\end{array}$ & $\begin{array}{c}\text { Projections } \\
\text { survivants } \\
\text { Femmes }\end{array}$ & $\begin{array}{l}\text { Projection } \\
\text { survivants } \\
\text { totale }\end{array}$ \\
\hline LBP & 605 & 216 & $70 \%$ & $45 \%$ & 423,5 & 97,2 & 520,7 \\
\hline CEsophage & 82 & 23 & $15 \%$ & $28 \%$ & 12,3 & 6,44 & 18,74 \\
\hline Estomac & 133 & 94 & $35 \%$ & $38 \%$ & 46,55 & 35,72 & 82,27 \\
\hline Colon-rectum & 459 & 487 & $65 \%$ & $70 \%$ & 298,35 & 340,9 & 639,25 \\
\hline Foie & 100 & 51 & $20 \%$ & $32 \%$ & 20 & 16,32 & 36,32 \\
\hline Pancréas & 94 & 82 & $15 \%$ & $35 \%$ & 14,1 & 28,7 & 42,8 \\
\hline Larynx & 116 & 37 & $59 \%$ & $72 \%$ & 68,44 & 26,64 & 95,08 \\
\hline Poumon & 633 & 439 & $17 \%$ & $30 \%$ & 107,61 & 131,7 & 239,31 \\
\hline Plèvre & 4 & 5 & $23 \%$ & $46 \%$ & 0,92 & 2,3 & 3,22 \\
\hline Vessie & 89 & 28 & $80 \%$ & $55 \%$ & 71,2 & 15,4 & 86,6 \\
\hline Rein & 264 & 140 & $75 \%$ & $81 \%$ & 198 & 113,4 & 311,4 \\
\hline $\begin{array}{l}\text { Système } \\
\text { nerveux central }\end{array}$ & 409 & 335 & $47 \%$ & $55 \%$ & 192,23 & 184,25 & 376,48 \\
\hline $\begin{array}{l}\text { Lymphome } \\
\text { non hodgkin }\end{array}$ & 743 & 426 & $70 \%$ & $74 \%$ & 520,1 & 315,24 & 835,34 \\
\hline Myélome & 49 & 36 & $69 \%$ & $70 \%$ & 33,81 & 25,2 & 59,01 \\
\hline $\begin{array}{l}\text { Leucémies } \\
\text { aigues }\end{array}$ & 316 & 267 & $43 \%$ & $54 \%$ & 135,88 & 144,18 & 280,06 \\
\hline Col de l'utérus & & 1181 & & $80 \%$ & 0 & 944,8 & 944,8 \\
\hline L'utérus & & 129 & & $85 \%$ & 0 & 109,65 & 109,65 \\
\hline Ovaire & & 351 & & $71 \%$ & 0 & 249,21 & 249,21 \\
\hline TOTAUX & 4096 & 4327 & $52,32 \%$ & $64,42 \%$ & 2142,99 & 2787,25 & 4930,24 \\
\hline
\end{tabular}

\subsection{Un impact minime pour les assureurs}

Dans son dernier rapport [7], la Fédération Française des Sociétés d'Assurances indique que pour l'année 2014, les cotisations perçues par l'ensemble des sociétés d'assurances françaises et étrangères opérant en France au titre des assurances de crédits, de la protection juridique et des prestations d'assistance s'élèvent à 5,6 milliards d'euros tandis que les prestations versées au même titre s'élèvent à 2,6 milliards d'euros. 
Comparativement à l'ensemble des cotisations (dont le montant était de 200 milliards d'euros dont 51,1 pour l'assurance des biens et des responsabilités; dont fait partie l'assurance des prêts) et des prestations (qui s'élèvent à 155,2 milliards dont 36,1 milliards qui nous intéressent), l'assurance emprunteur reste un poste marginal de l'activité des assureurs. A fortiori, l'assurance de 98 millions d'euros de prêts immobiliers supplémentaire ne représenterait qu'une infime modification de ces cotisations et prestations.

\subsection{Un effet d'appel d'air limité.}

Le passage du droit à l'oubli dans un délai de 15 à 5 ans pour les cancers de bon pronostic aurait également un effet "d'appel d'air". En d'autres termes, un certain nombre des personnes qui n'auraient pas pu emprunter sans déclarer leur cancer vont se retrouver dans la possibilité de le faire.

Plus précisément, ces individus sortis des traitements il y à 5 ans et plus, pour correspondre toujours à la classe d'âge susceptible d'emprunter sans surprime d'assurance (les moins de 55 ans) en 2017 doivent avoir été diagnostiqués avant 2012. De plus, ils doivent appartenir à une classe d'âge différente selon l'année du diagnostic.

La borne supérieure de cette classe d'âge est donnée par la différence entre l'âge maximum d'emprunt sans surprime (54 ans) et le nombre d'années écoulées en 2017 depuis le diagnostic. Il est à noter que les individus mineurs au moment du diagnostic sont exclus des classes d'âges quelle que soit l'année du diagnostic (entre 2002 et 2012) dans la mesure où le droit à l'oubli leur est déjà accordé au bout de 5 ans.

Nous avons donc utilisé la pyramide des âges au 1er janvier 2015 afin de réaliser une extrapolation non linéaire des nombres de cancer diagnostiqués entre 2002 et 2011 sur les classes d'âge qui pourraient être concernées par cet "appel d'air".

Nous avons alors appliqué les taux de survie brute actuels à chacun de ces cancers. Cela constitue une estimation grossière du nombre potentiel de personnes restant en vie en 2017 et qui pourraient profiter de l'abaissement de la durée d'accès au droit à l'oubli à 5 ans lors de sa mise en place éventuelle en 2017 pour demander un crédit immobilier.

En 2017, le nombre maximum estimé de patients diagnostiqués pour un cancer de bon pronostic entre 2002 et 2012 encore en vie s'élèverait à 168 200. Par conséquent, le nombre de demandes potentielles de 
crédits immobiliers pourrait être au maximum de 6112 portant à 568,9 millions d'euros le montant maximum des crédits ainsi demandés à la mise en application d'une diminution du délai de droit à l'oubli, si on considère le montant moyen actuel des prêts immobiliers (93,1 milliers d'euros). Encore une fois, ce montant supplémentaire de crédits à assurer ne représente qu'une très faible augmentation par rapport au montant total des prêts immobiliers assurés en 2015.

TABLEAU 3 Estimation du nombre maximum d'individus diagnostiqués pour un cancer de bon pronostic entre 2002 et 2012 encore en vie en 2017

\begin{tabular}{l|c|r|r}
\hline $\begin{array}{l}\text { Localisation } \\
\text { du cancer }\end{array}$ & $\begin{array}{r}\text { Nombre de cancers diagnostiqués } \\
\text { entre 2002 et 2012 dont } \\
\text { les patients auraient } \\
\text { entre 18 et 54 en 2017 }\end{array}$ & $\begin{array}{r}\text { Taux de } \\
\text { survie brute } \\
2015\end{array}$ & $\begin{array}{c}\text { Survivants } \\
\text { potentiels } \\
\text { en 2017 }\end{array}$ \\
\hline Sein & 91639,37 & $79,00 \%$ & 72395,1 \\
\hline Testicule & 15568,83 & $93,00 \%$ & 14479,0 \\
\hline Prostate & 2520,07 & $67,00 \%$ & 1688,4 \\
\hline Thyroïde & 24298,78 & $90,00 \%$ & 21868,9 \\
\hline Mélanome & 25936,52 & $78,00 \%$ & 20230,4 \\
\hline Lèvres & 52882,45 & $71,00 \%$ & 37546,5 \\
\hline & & Total & $\mathbf{1 6 8 2 0 8 , 5}$ \\
\hline
\end{tabular}

\section{CONCLUSIONS}

L'étude, fondée sur des chiffres fournis par des instituts et organismes reconnus par les professions, a clairement démontré que le surcroît de risque à assurer lors de la souscription à un crédit immobilier par les personnes atteintes d'un cancer de bon pronostic 5 ans après le diagnostic est très minime, pour ne pas dire quasi nul. En tout état de cause, il restera marginal sur le marché des assurances de prêts immobiliers.

Ce constat est d'autant plus vrai que les chiffres les plus défavorables ont été mis en avant dans cette synthèse. Ainsi, si on considère l'hypothèse plus probable d'un nombre de personnes survivantes à 5 ans de 15 892, l'augmentation du montant des crédits à assurer liée à la réduction du droit à l'oubli à 5 ans est au maximum de 8 pour 10000 . 
Similairement, le passage à 10 ans du droit à l'oubli pour les cancers de pronostic intermédiaire ou mauvais aurait des conséquences marginales sur le montant des prêts à assurer avec une estimation à moins de 200 demandes de crédits immobiliers par an.

Même en cas d'évolution défavorables à l'étude de certaines données (augmentation du nombre d'intentions de demandes de crédits, augmentation du montant moyen des crédits, diminution des taux de survie à 5 ans), les surcroîts de risque à assurer en raison de la mise en place d'un droit à l'oubli à 5 ans resteraient minimes.

D'autre part, le surcroît maximum de crédits à assurer lors de la mise en application du passage du délai de droit à l'oubli de 15 à 5 ans, lié à l'opportunité offerte à une cohorte de patients diagnostiqués entre 2002 et 2012 ne représente qu'une augmentation de 0,3\% du montant total des prêts immobiliers à assurer.

Enfin, si les études concernant les risques de développer un second cancer sont récentes et n'ont été publiées pour la première fois que récemment [3], elles encouragent néanmoins à penser que les cancers de bons pronostics sont également ceux pour lesquels les patients ont un risque à peine plus élevé que le reste de la population de développer un autre cancer. En effet, pour chacun d'entre eux, les patients ont un risque moins de deux fois plus élevé que l'ensemble de la population de développer un second cancer. Comparativement aux patients atteints de cancer de moins bon pronostic qui peuvent présenter un risque jusqu'à 4 à 6 fois plus élevé, il est clair que l'assurance des patients atteints de cancer de bon pronostic 5 ans auparavant ne s'éloigne pas de manière spectaculaire de celle du reste de la population.

\section{REMERCIEMENTS}

L'auteur tient à remercier l'association ROSE et tout particulièrement Céline Lis-Raoux, sa présidente, pour lui avoir offert l'opportunité de s'ouvrir à ce sujet passionnant et pour lui avoir permis de communiquer les résultats de cette étude dans le cercle académique. 


\section{RÉFÉRENCES}

[1] M. Mouillart, "27ème rapport annuel», Observatoire des Crédits aux Ménages, 2015.

[2] J.-M. Vilon, M. Mouillart, "L'observatoire du financement des marchés résidentiels", 9ème édition, Crédit logement - Institut CSA, 2015.

[3] P. Grosclaude, L. Remontet, A. Belot, A. Danzon, N. RasamimananaCerf, N. Bossard, "Survie des personnes atteintes de cancer en France 1989-2007 Étude à partir des registres des cancers du réseau Francim", Institut de veille sanitaire, Registre des cancers Francim, Hôpitaux de Lyon, Institut National du cancer, 2013.

[4] N. Vongmany, "Les cancers en France, Les Données", Edition 2014, Institut National du Cancer, 2015.

[5] A. Monnereau, L. Remontet, M. Maynadié, F. Binder-Foucard, A. Belot, $\mathrm{X}$. Troussard, N. Bossard, "Estimation nationale de l'incidence des cancers en France entre 1980 et 2012 - Étude à partir des registres des cancers du réseau Francim; Partie 2 - Hémopathies malignes ", InVS, INCa, 2013.

[6] A. Belot, "Estimation nationale de l'incidenceet de la mortalité par cancer en France entre 1980 et 2005", Institut de veille sanitaire (Département des maladies chroniques et traumatismes) et Hospices civils de Lyon, 2008.

[7] LCOMFI, Rapport annuel 2014, 2015, Fédération Française des Sociétés d'Assurances.

[8] Jégu J, Colonna M, Daubisse-Marliac L, Trétarre B, Ganry O, Guizard A-V, et al., The effect of patient characteristics on second primarycancer risk in France., BMC Cancer 2014, 2014.

\section{NOTES}

1. Université de Strasbourg, CNRS, BETA UMR 7522, F-67000 Strasbourg, FranceMembre de la chaire UNESCO «Pratiques journalistiques et médiatiques»

2. Impliquant les pouvoirs publics et les professionnels de l'assurance, les associations de consommateurs et les associations représentants les personnes malades ou handicapées.

3. Institut National du Cancer 